Satisfaction in Choice as a Function of the Number of Alternatives:

When "Goods Satiate" but "Bads Escalate" *

Elena Reutskaja \& Robin M. Hogarth

November 2005

\footnotetext{
* Elena Reutskaja is a $\mathrm{PhD}$ candidate in the Graduate Program of Economics, Finance and Management at Universitat Pompeu Fabra, Ramon Trias Fargas 25-27, 08005, Barcelona, Spain. (Tel: +34 93542 1621, Fax + 34 93542 2533, e-mail: elena.reutskaja@upf.edu).

Robin M. Hogarth is ICREA Research Professor at Universitat Pompeu Fabra, Department of Economics and Business, Ramon Trias Fargas 25-27, 08005, Barcelona, Spain. (Tel: +34 93542 2561, Fax + 3493 542 1746, email: robin.hogarth@upf.edu).

The authors are grateful for helpful comments on this research from Elizabeth Cowley, Barbara Fasolo, Ralph Hertwig, Barbara Kahn, Antonio Ladrón de Guevara, Rosemarie Nagel, Albert Satorra, and Marc Vanhuele. The research was financed partially by a grant from the Spanish Ministerio de Educación y Ciencia (to R. M. Hogarth).
} 


\begin{abstract}
People often prefer to choose from small as opposed to large sets of alternatives. We propose that satisfaction from choice is an inverted U-shaped function of the number of alternatives. This proposition is derived theoretically by considering the benefits and costs of different numbers of alternatives and is supported by three experimental studies. Because, in large sets, the perceptual costs of processing alternatives varying in shape are greater than for alternatives varying in color, we also predict and demonstrate greater satisfaction from choosing from the latter. We further show that the "satisfaction function" is affected by gender and cultural background.
\end{abstract}

Keywords: Consumer choice; perception of variety; tyranny of choice; visual perception; cultural differences.

JEL classification: D12, M10, M31. 
A major tenet of classic economic theory is that more choice is always desirable.

However, there is a growing body of research suggesting that extensive choice may be costly and demotivating to consumers (Desmeules 2002, Dhar 1997; Iyengar and Lepper 2000;

Loewenstein 1999;. Schwartz 2000; Shafir Simonson and Tversky 1993). So how much choice is enough? In advanced modern societies this is an important issue that affects not only what firms can and should bring to market but also possibly even the mental health of the consuming public (Schwartz 2004).

In this paper, we explore how satisfaction from choice varies as a function of set size or "variety," that is, the number of alternatives faced. By satisfaction we mean satisfaction both from the product chosen and from the process of choice. In doing so, we point out that the set sizes examined in previous studies favoring choice are typically limited (up to six options) while the sets claimed to be demotivating are typically large (24-30 options) (Iyengar and Lepper 2000; Kahn and Wansink 2004; Rolls et al. 1981). Curiously, in previous research little attention has been paid to the levels of satisfaction consumers experience when they face intermediate numbers of alternatives (e.g., between 10 and 20 options). In addition, there is a surprising lack of theoretical insight into why different levels of set sizes might induce different levels of satisfaction.

This paper extends previous studies to different numbers of alternatives in choice sets limited, intermediate, and extensive. Moreover, we build upon the theoretical insights of Coombs and Avrunin (1977) to suggest that satisfaction in choice is an inverted U-shaped function of the number of alternatives in a set.

More specifically, consider the benefits and costs of increasing the number of alternatives in a set. As the number of alternatives increases, so do the benefits (e.g., more options become 
available); however, so do the costs of making a choice (e.g., time, costs of information processing, and so on). Now define satisfaction as equivalent to net benefits (i.e., benefits less costs) and picture how this varies as a function of the number of alternatives. If costs increase faster than benefits - or "goods satiate" while "bads escalate" - it follows that satisfaction from choice will be an inverted U-shaped function of the number of alternatives in a set, as illustrated in figure 1. ${ }^{1}$ Given this insight, we present two experiments that test whether "satisfaction functions" do indeed have this single-peaked shape.

Insert Figure 1 about here

Our theoretical framework implies that changes in information processing costs will change the position of the peak of the "satisfaction function." For example, holding benefits constant, lower costs will shift the peak to the right whereas greater costs will shift it to the left. Thus, since the manner in which information is presented can affect processing costs, we also examine this variable by manipulating the visual presentation of choice alternatives.

Extrapolating from findings concerning limitations on the human visual system, and the degree of comfort - and hence psycho-physical cost - that people experience when confronted with different types of visual environments (Filin 2000), we predict that, when offered large sets, consumers will experience higher satisfaction when alternatives differ in color as opposed to shape.

Existing literature suggests that consumers' feelings about choice decisions may also reflect individual differences (Schwartz et al. 2002; Iyengar and Lepper 1999; Desmeules 2002).

\footnotetext{
1 Coombs and Avrunin's (1977) analysis of single-peaked functions is discussed within the context of preferences. However, their mathematical reasoning can be applied to any process that has the appropriate structure.
} 
We therefore consider how individual characteristics such as gender and cultural background might affect satisfaction from variety.

The first part of the paper provides the theoretical framework for the empirical studies. The second presents the results of three experiments. Experiment 1 explores the shape of the satisfaction function from choice, and examines the effect of visual characteristics of choices on satisfaction. Experiment 2 is a replication of experiment 1 in two Eastern European countries. Experiment 3 investigates whether and under what conditions customers are more attracted to assortments where alternatives differ in color but not in shape. The paper concludes by discussing the implications of the results and possible topics for future research.

\section{MOTIVATION AND THEORETICAL FRAMEWORK}

Choice Variety and Single-Peaked Functions.

As noted above, we propose that the nature of satisfaction as a function of set size has a structure equivalent to that of the single-peaked preference functions analyzed by Coombs and Avrunin (1977). There are "goods" (benefits) and "bads" (costs) of choice and satisfaction from variety (number of choice alternatives) is the difference between the two.

Variety does indeed have benefits. It increases the likelihood of satisfying diverse consumers; it promotes competition by driving price down and quality up; it gives people the feeling of autonomy and self-control, thereby facilitating intrinsic motivation (Loewenstein 1999, Ryan and Deci 2000, Zuckerman et al. 1978). It might also add to the happiness of consumers who enjoy shopping by creating positive affective experiences; and actual and 
perceived variety may increase consumption (Desmeules 2002, Kahn and Wansink 2004, Rolls et al. 1981).

On the other hand choice also induces costs. First, large choice offerings may be undesirable due to limited human information processing abilities. In a classic paper, Miller (1956) argued that human abilities to receive, process, and remember information are limited. The "span of absolute judgment," or the greatest amount of information that a human can process accurately, is shown to be surprisingly low for uni-dimensional judgments: for tones it is as low as six, for taste stimuli, four, and for visual positioning, the largest channel capacity, 10 to 15 . Therefore, we argue that when offered large choice sets, consumers may not be able to process all the information accurately, that is, the cost of information processing will increase and escalate with the number of alternatives.

Loewenstein (1999) suggests that extensive choice also imposes time, error, and psychic costs. When facing extensive offerings, customers may experience what Schwartz (2000) has named the "tyranny of freedom." Close consideration of the alternatives may also induce "attachment" to the options in the choice set such that people feel the "loss" of the items they have not chosen (Carmon, Wertenbroch, and Zeelenberg 2003). Also, the more options foregone, the greater the post-choice discomfort experienced. Moreover, increasing the number of alternatives in the choice set, having items with similar attractiveness as well as difficulty in deciding may make people defer choice or simply choose a default option (Shafir et al 1993; Dhar 1997).

Iyengar and Lepper (2000) have demonstrated that large assortments may be demotivating. In their field study, although customers were more attracted to an array with 24 as opposed to six different jams, they purchased less from the larger set. In addition, other studies 
by these researchers have shown that people consume more and even perform better on intellectual tasks (writing essays) when facing limited rather than extensive sets of options.

In sum, the evidence demonstrates that the provision of choice has both "goods" and "bads." When the set size increases, the benefits of choice rise, but satiate, whereas the costs of choice increase, and escalate. ${ }^{2}$ More formally, we state:

H 1: The size of the choice set affects consumer satisfaction. Satisfaction is an inverted U-shape function of the number of alternatives in the choice set.

Visual Organization and Presentation of the Assortment Variety.

Several studies suggest that the manner in which assortments are presented can affect decisions, especially, when the choice set is large. For example, some time ago Miller (1956) noted that the organization of information into "chunks" or sequences facilitates information processing. More recently, Kahn and Wansink (2004) claimed that the organization and entropy of an assortment affects perceived variety thereby influencing consumption. They further argued that when perceived variety increases, so does consumption. For large choice sets, perceived variety is higher in organized sets; for smaller sets, it is greater in disorganized sets.

Huffman and Kahn (1998) investigated how to present large assortments to consumers without decreasing satisfaction. They demonstrated that, for high-variety assortments, consumers were more satisfied (in terms of learning their own preferences), perceived less complexity, and were more willing to make choices when assortments were presented in attribute- rather than alternative-based formats.

\footnotetext{
${ }^{2}$ Desmeules (2002) has also suggested that, when evaluating alternatives cognitively, the consumption experience might have an inverted U-shaped relationship across set size. However, his proposition was neither formalized nor tested empirically.
} 
We suggest that satisfaction from variety is affected by the visual presentation of sets in that this affects information processing costs. For example, noting the implications of limitations in human visual abilities, Filin (2000) argues that individuals experience a feeling of discomfort and dissatisfaction in two "poorly organized" visual environments: "aggressive" environments (i.e., those with a great concentration of similar elements) and "homogeneous" environments (i.e., those with monotonic visual scenes, like plain white walls).

In our work, we consider the effect of two visual qualities - color and shape. ${ }^{3}$ We suggest that if an assortment is large and the alternatives in the set differ only in form, the assortment has a "monotonic" look such that the consumer faces a "homogeneous" visual environment that imposes high processing costs, and consequent discomfort. Provision of colors, however, may resolve the problem of monotonicity by making the items more distinct thereby reducing the costs for the human visual system.

Indeed, Spring and Jennings (1993) claim that hue is recognized pre-attentively, while complex shape is a non-preattentive stimulus that requires more time to be processed. Thus, to the extent that hue is a pre-attentive stimulus, its detection should be insensitive to the size of the search set in which it is presented whereas this would not be the case for complex shapes (Spring \& Jennings 1993). Moreover the attentional costs of presenting a non pre-attentive stimulus like shape should be particularly high in larger sets.

We therefore propose that, when the set of alternatives is large, the cost of choice is higher for assortments with alternatives differing in shape than for those differing in color. As a result, we expect consumers to be more satisfied when they face large assortments with options

\footnotetext{
3 We use the words "form" and "shape" interchangeably. Our purpose here is not to determine how visual characteristics of separate objects influence consumer decisions but rather how the visual characteristics of the entire set affect satisfaction from variety. Individual preferences for colors and forms are not, therefore, a subject of the current paper.
} 
that differ in color as opposed to shape. In other words, the peak of the satisfaction function (see above) for colors will lie to the right of that for shapes.

However, when the choice set is small (i.e., within human information processing limits), the negative impact on the human visual system should not be significant and we expect no such difference to occur. More formally, we state:

H 2: Visual presentation of assortment affects consumers' satisfaction. Consumers experience higher satisfaction when the alternatives in large choice sets are different in color but not in form. However, for small choice sets, consumers are equally satisfied when alternatives are presented in either different colors or forms.

\section{EXPERIMENT 1}

The aim of experiment 1 was to explore the shape of the satisfaction function from variety and examine the impact of visual presentation of assortment on satisfaction. In this laboratory experiment, participants were asked to assume that they were shopping for a gift box to pack a present for a friend and were given a picture of either a small, medium or large sized set of gift boxes. The participants had to choose one gift box and report their levels of satisfaction from the choice.

Method

Assortment. The gift boxes differed from each other on two visual attributes: color and/or form. The sets where the gift boxes were of the same form and different colors will be referred to 
as "SFDC" sets, of the same color and different forms will be called "SCDF" sets, and of different colors and different forms will be referred to as "DCDF" sets. We will call SFDC and SCDF sets "simple" sets, as the items in these sets differ from each other on only one attribute, while the DCDF sets will be referred to as "complex" sets, where options differ on two attributes.

Previous research demonstrates that entropy and organization affect perceived variety (Kahn and Wansink 2004). To control for entropy, no choice sets contained identical alternatives. To control for organization, all assortments presented to the participants were organized (e.g., by shading of colors). Moreover, colors and forms in the sets were varied across the experimental stimuli to control for color and form preferences. The boxes did not contain anything and, except for visual characteristics, were said to be identical.

Procedure. Participants were randomly divided into 12 experimental groups formed by crossing two between-subject factors - number of choice options with four levels (five, 10, 15 or 30), and three types of assortments, SFDC, SCDF, and DCDF.

Participants had to examine pictures with gift boxes and state which they would buy to pack a present for a friend. After stating their choices, they answered a paper-based questionnaire, evaluating their satisfaction from the choice, and providing demographic characteristics.

Participants. The 120 participants were students and professors at several universities in Barcelona. All spoke English; 43\% were Spanish and most of the others came from EU 
countries. Average age was 23.7 years, and $53 \%$ were female. They received no financial remuneration.

Dependent measures. We define a satisfied customer as one who both likes the item she has chosen and enjoyed the process of choosing. Participants were therefore asked two questions assessing their satisfaction from the alternative they decided to choose (specifically, "How much do you like the gift box you decided to pick?" and "How different/similar is the box you chose from the 'ideal' box you would like to purchase for your friend?"), and two questions evaluating their pleasure from the decision making process (specifically, "How much did you enjoy making the choice (the decision process)?", "Did you find it difficult to make your decision of which gift box to purchase?"). Responses were provided on a Likert scale ranging from one ("not at all") to

\section{0 (“extremely"). ${ }^{4}$}

To evaluate whether participants felt they had been offered too many, too few, or the right amount of options, the respondents were also asked: "Do you feel you had the right amount of options to choose from?" Responses were provided on a nine-point Likert scale with "1" meaning, "No, I had too few choice options", "5" meaning, "Yes, I had just the right number of choice options", and "9", meaning, "No, I had too many choice options".

It is worth noting that most of the measures used in this experiment were similar to those used by Iyengar and Lepper (2000) in their study 3 which motivated the current research.

\footnotetext{
${ }^{4}$ The Spanish educational system uses a 10-point system for evaluating the performance of students. As the majority of participants were studying or working at Spanish universities, we believe that a 10-point scale was the easiest for them to interpret and use. For the question about the "ideal gift box", "1" meant "The ideal gift box would be very different from the box I chose now", and "10" meant "The box I chose is the ideal one."
} 
Results

Satisfaction from the choice function. As shown by four measures of satisfaction in figures $2-5$, the results of experiment 1 strongly support our first hypothesis: satisfaction is an inverted U-shape function of the number of alternatives in the set.

Insert table 1 and figures $2-6$ about here

In terms of statistical tests, table 1 summarizes ANOVA output indicating that the size of the choice set significantly affects consumer satisfaction for all five dependent measures. Statistical tests of the nature of these differences (i.e., whether satisfaction functions have inverted U shapes) are presented in appendix A. This shows, for example, that for "satisfaction from the gift box picked" (figure 2), the mean satisfaction from 10 options (8.5) is significantly greater than both those for five and 15 options (i.e., 7.0 and 7.7, respectively), and that satisfaction for 15 options exceeds that for 30 (i.e., 7.7 vs. 7.1 ).

Overall, and consistent with our expectations, participants reported lower satisfaction with choice from limited (five) and extensive (30) options, and higher satisfaction from mediumsized sets (10 and 15 options). The 10-option set was found to be the most satisfying. When encountering such sets, respondents also felt the box chosen was the most satisfying, the most similar to "ideal," and that the decision process was the most enjoyable. Results revealed that as set size increased, difficulty of choosing also increased, though not significantly. Participants further believed that the "right number of options" was 10 or 15 - see figure $6 .{ }^{5}$ The 30 -option set was considered to be overwhelming, while the five-item set was perceived as offering too little choice.

5 Recall that on this scale five was "ideal" with one being "too few" and nine "too many." 
Gender. ANOVA revealed significant gender differences in satisfaction from the alternative chosen measures (controlling for the set size) - for details of statistical tests, see appendix B. Women reported higher satisfaction from the box they decided to pick and, compared to men, believed that the box they had chosen was more similar to "ideal" over the whole range of set sizes. However, no significant gender effects were found for the other dependent variables; nor were the interactions for gender and set size significant for any of the dependent variables.

Complexity. According to Iyengar and Lepper (2000), previous studies have demonstrated that people prefer to examine fewer alternatives when both the set size and information about alternatives increase. In this experiment, the effects of complexity of visual attributes could also be tested because we had sets with both one-dimensional (simple) and two-dimensional (complex) items. ANOVA (see appendix C) showed that participants facing simple assortments were significantly more satisfied both from the gift box picked and from the decision process than those encountering complex choice sets (controlling for the set size). Interactions for set size and complexity were not significant for any of the dependent variables.

Visual presentation. Experiment 1 was also designed to test whether two visual qualities of the choices - color and form - affect satisfaction from variety. We therefore analyzed the responses of the 80 participants who faced SCDF and SFDC sets.

The analysis (using ANOVA) supported our second hypothesis. Participants facing large sets (i.e., 30 options) with alternatives that differed in color reported significantly higher satisfaction from the box they decided to pick $[F(1,72)=10.93, p=.002]$ than those who 
encountered large sets where alternatives differed in form (see figure $7 \mathrm{a}$ and $\mathrm{b}$ ). For the small sets, however, this difference was not significant $[F(1,72)=0.95, p=.334 ; F(1,72)=3.06, p=$ $.084 ; F(1,72)=0.95, p=.334$ for five-, 10 - , and 15-option sets respectively]. Moreover, the participants facing SFDC assortments were significantly more satisfied from the process of choosing than those who encountered SCDF sets over the entire range of sets sizes $[F(1,75)=$ $4.15, p=.045]$.

Insert figure 7 about here

Visual format also affected participants' beliefs about the right number of options in the set. When alternatives in the sets differed in color, the participants believed that 15 - or even 30 option sets contained "about the right number of options" $[F(1,72)=1.65, p=.203 ; F(1,72)=$ $1.65, p=.203$ respectively]. However, when the choices were different in form, 30 options were viewed as "more than the right amount" $[F(1,72)=26.40, p=.000]-$ see figure $7 \mathrm{c}$. There were no significant differences in the other two dependent measures due to different visual presentations of the choice sets.

\section{Discussion of Experiment 1}

Experiment 1 provided empirical evidence for differences in satisfaction due to the number of available alternatives. Satisfaction was shown to be an inverted U-shape function of the number of options in the set. The function increases when the benefits of choice are higher than the costs, and it decreases when the "bads" become greater than the "goods." However, the peak of the function, or the highest satisfaction level, may not be a single point, but a range of alternatives, that are equally satisfying. For example, in our work the satisfaction from the 
decision process function did not decrease significantly after the 10-option set. We believe that 10-, 15-, and 30-option sets all belong to "the optimum" of this function, and an "overwhelming" number of alternatives was not reached in this case. On the other hand, when the set becomes even larger and the costs outweigh the benefits of choice, we predict that satisfaction from the decision process will fall significantly. To test this proposition, however, further experimental research is needed.

Experiment 1 also demonstrated that satisfaction is a matter of the visual layout of alternatives. As expected, people experience higher satisfaction when the alternatives in large choice sets differ in color as opposed to form, whereas for the small sets they may be equally satisfied from alternatives presented in different colors or different shapes. We believe that due to particularities of the human visual system discussed above, the costs of information processing are greater for the sets with alternatives differing in shapes than for those with options varying in color especially when the choice set is large (otherwise we would find significant differences across the entire range of the sets). Therefore, for the former, the resulting satisfaction function reaches its peak and starts to fall earlier (i.e. the peak is shifted to the left) than for the latter.

In addition, we suggest that introduction of colors may also increase perceived variety, which, in turn, should increase the benefits of choice for a consumer, that is, by shifting the peak of the satisfaction function to the right. In a study on food intake, Rolls et al. (1981) found that participants consumed more yogurt when three yogurts - differing in taste, color and texture were presented than when just one yogurt flavor was offered. Interestingly, when three yogurts were of different flavors, but of the same appearance, no enhancement of food intake was found in comparison with the offering of just one flavor. The authors do not propose any explanation for this result. Our findings, however, can be relevant by suggesting that the presentation of 
alternatives in different colors increases the perceived benefits of variety and thus its attractiveness.

The findings concerning presentation of variety may have important implications for marketing and, in particular, advertising and merchandizing. The results suggest that by presenting alternatives of large assortments in different colors retailers may create "comfortable" visual environments for customers thereby influencing their satisfaction from choice. As a result, consumers may be able to obtain high benefits from wide assortments without experiencing overwhelming costs, and in turn, be able to process large amounts of information without losing satisfaction.

Experiment 1 also suggests that the function of satisfaction "from the product chosen" may depend on gender. For males the function lies below that for females. Why this occurred is unclear. Several explanations come to mind.

First, at a cognitive level, there is some evidence that women are used to paying more attention to detailed information than men and this habit might be thought to lower the perceived costs of information processing in consumer-related tasks (cf., Meyers-Levy and Maheswaran 1991; Meyers-Levy 1998). Second, at a social level, females may obtain more pleasure from "shopping" and "buying" and thus care more about items such as gift boxes than males. Third, Schwartz et al. (2002) have argued that people can be thought of as "maximizers" or "satisficers" and that the former experience less satisfaction with their decisions than the latter. Therefore, if men tend to be maximizers, and women satisficers, the levels of satisfaction with choice would differ by gender. However, whether males were indeed maximizers and females were satisficers was not explicitly tested in experiment 1 and requires further exploration. 
One could also argue that gender effects are product dependent: for a different product category (e.g., beer or mobile phones) one might find a reversed effect. Therefore, whether gender effects can be generalized across different conditions remains unclear from the current experiment and is an interesting topic for further research.

The results of experiment 1 also showed that some sub-samples of individuals with similar cultural background reported similar satisfaction levels (not reported). Whether this was a coincidence or a general trend was impossible to detect due to limited sub-sample sizes. However, recent studies do suggest that choice may reflect cultural differences. Iyengar and Lepper (1999), for example, demonstrated cultural effects on intrinsic motivation: EuropeanAmericans are more intrinsically motivated by personal choice whereas Asian-Americans may prefer to have choices suggested to them by "valued ingroup members". We therefore took the opportunity to replicate experiment 1 in an Eastern European sample and to compare results with the data obtained in Western Europe (see below).

\section{EXPERIMENT 2}

The goal of experiment 2 was to replicate experiment 1 in Eastern European countries and thereby also investigate possible cultural influences in the experience of satisfaction. The design was identical to that of experiment $1 .^{6}$ Participants of experiment 2 were 120 students and professors from several universities in two Eastern European countries: Belarus (85\%) and Ukraine (15\%). Average age of the participants was 21 years, and 53\% of the participants were males. They were not remunerated for their participation.

\footnotetext{
6 The studies in Eastern Europe were, however, conducted in Russian.
} 
Results

Satisfaction from choice. In accordance with hypothesis 1, the satisfaction function of the Eastern European participants had an inverted U-shape similar to their Western European counterparts (see figures $2-5$, table 1 and appendix A). At the same time, a Chow test indicated a significant difference in satisfaction from the gift box picked between the Western and Eastern European samples $[F(4,236)=190, p=.000]$. Participants from Belarus and Ukraine reported the highest satisfaction from 15- and 30-option sets whereas Western Europeans were most satisfied from medium-sized assortments. The peak of the function, therefore, was shifted toward the greater number of alternatives in the Eastern European sample, assortments with 15 options being seen as the most satisfying ${ }^{7}$. Interestingly, the participants also reported the lowest difficulty levels when choosing from such sets, and considered that the 15-option set included exactly the "right number of boxes."

Gender and complexity. As in the Western European sample there were significant gender and complexity effects for some dependent variables in the Eastern European sample (see appendices B and C). Eastern European females reported significantly higher satisfaction levels than men from both the box picked and the decision process. Across all set sizes, satisfaction from the box picked was lower for participants facing complex as opposed to simple sets.

Visual presentation. ANOVA revealed that visual presentation of the sets affected satisfaction of the Eastern European participants as well. Consistent with the findings of experiment 1 (and hypothesis 2), Eastern European participants reported higher satisfaction both

7 The overall means of satisfaction from the product were not different for the two samples $(t=0.084, p=.093)$. 
with the gift box picked $[F(1,72)=4.02, p=.049]$, and with the decision process $[F(1,72)=$ $3.13, p=.081$ ], when facing large sets (30 options) where alternatives differed in color as opposed to form. In addition, when facing SFDC sets, participants felt they had fewer options than when encountering the same sized SCDF assortments $[F(1,75)=8.26, p=.01]-$ see figure 8.

Insert figure 8 about here

Discussion of Experiment 2

Experiment 2 provided support for hypothesis 1 in a different cultural sample, that is, in Eastern as opposed to Western Europe. At the same time, the results suggest that cultural background can affect perceptions of the costs and benefits of variety in choice. Participants from Eastern Europe sought greater variety than their Western counterparts and were more satisfied with larger choice sets, that is, the peak of satisfaction function for Western Europeans lies to the left of that for Eastern Europeans.

The reason for this finding is not apparent. Given the comparative paucity of variety in shops in Eastern Europe until fairly recently, one might have imagined the contrary result, that is, Eastern Europeans would have been more satisfied with less variety. On the other hand, perhaps - by some unknown mechanism - relative lack of variety induces a desire for more.

We speculate briefly about this latter finding. First, people from Western societies may take the possibility of making choices for granted, and thus value variety less than those who lacked such opportunities for a long time. In contrast, people from regions where choice had 
been limited in the recent past might imagine that "ideal" offerings should be large, and even now might feel that they do not have as much choice as their Western counterparts. They are therefore motivated to seek as much choice as possible.

Second, precisely because people from Eastern Europe experienced scarcity in choice, they were required to face more decisions in everyday life: where, when and how to find necessary items unavailable in shops, and what to do if unable to find them. In other words, one could argue that daily life in Eastern Europe was more complex than that in the West, and being used to this, Eastern Europeans are capable of handling greater variety and complexity than those in the West.

As noted above, however, these arguments are speculative and should be subjected to empirical tests. Whatever the reason, it is important to note that assortments in Eastern European countries have been increasing dramatically in recent years. Thus, when choice offerings in Eastern Europe become comparable to those in the West, Eastern Europeans may well perceive variety in a similar manner to Western Europeans, and the desire for more extensive offerings might disappear. At the same time, the findings of experiment 2 suggest that the same marketing strategies concerning choice variety might lead to different results in different countries and that marketers should pay attention to the possibility of such differences.

Experiment 2 showed that visual presentation of assortment influences satisfaction levels of the participants from Western and Eastern European cultures in a similar way. In both experiments 1 and 2, participants reported significantly higher levels of satisfaction when the alternatives in the large choice sets were different in color but not in shape (hypothesis 2). However, does this mean that the sets with alternatives different in color are also more attractive for customers than those that vary in form? 
As a corollary to hypothesis 2 , therefore, we suggest that since visual "comfort" is more pleasing for the eyes (and less "costly" to process), one should also expect large sets with alternatives different in color to be more appealing than those that vary in form. Also - and once again - since information processing costs of small sets are not unduly taxing, we would not expect such effects with small sets. More formally, we state:

H 3: Visual properties of the set affect its attractiveness. More consumers are attracted to large assortments when alternatives differ in color as opposed to form. No such effects exist for small sets.

We conducted experiment 3 to test this hypothesis.

\section{EXPERIMENT 3}

Method

Procedure. The design of experiment 3 was similar to that of experiment 1 . The main difference was that, first, participants had to decide by themselves whether to choose from SCDF or SFDC assortments. Specifically, participants were told that there were two different stores selling gift boxes: "Shop A" offers gift boxes that vary in form (SCDF sets) whereas "Shop B" offers boxes that differ in color (SFDC sets). Choice sets presented to participants were identical to those of experiment 1. Participants were given two pictures: one representing the SCDF assortment, and the second showing the SFDC set. Both assortments offered to a particular individual were of the same size involving five, 10, 15, or 30 options. 
Participants were 48 undergraduate students at Universitat Pompeu Fabra in Barcelona. The average age was 19.2 and 26 were females. Participants were not remunerated. Groups of 12 participants were assigned at random to each of four groups evaluating the different-sized options.

First, participants had to choose in which shop - A or B - they would buy a gift box. Second, they were required to answer a questionnaire assessing their satisfaction and the difficulty of choosing between the two shops. ${ }^{8}$ After this, the participants were left with the picture representing the assortment of the shop they had selected. Third, participants followed the same procedure as in experiment 1 . They were asked to choose a gift box from the picture in their possession and complete the same questionnaire as in experiment 1.

Measures. First, we simply counted the numbers of participants who chose the different shops for the different set sizes. Second, we assessed participants' satisfaction from the assortment in each shop and the difficulty of choosing between them by asking "How much do you like the assortment of SHOP A?", "How much do you like the assortment of SHOP B?", and "How difficult was it for you to decide to which shop to go?" Responses were provided on a Likert scale raging from one ("Not at all") to 10 ("Extremely"). Third, satisfaction measures concerning choices of boxes were identical to those used in experiment 1.

Results

When facing medium or large choice sets (i.e., sets containing 10, 15 or 30 alternatives) 25 out of 36 participants preferred the assortment in shop B where boxes varied in color but not

\footnotetext{
8 Participants did not know that they would have to choose a box from this assortment afterwards.
} 
in shape thereby indicating that the former assortments are more attractive $[\mathrm{p}(\mathrm{x} \leq 11)=.025$, binomial test]. However, for small sets (five options), there was no significant difference (seven out of 12 participants found assortments with items different in forms more appealing, $[p(\mathrm{x} \leq 5)$ $=.387]$.)

Moreover, participants reported higher satisfaction levels from the assortments differing in color than in form for large and medium sets $(t=1.98, p=.056)$, and similar satisfaction levels for five-option sets $(t=0.98, p=.381)$. The average difficulty of choosing between shops was 4.7.

The means of the satisfaction variables from the choice of the gift boxes in experiment 3 were lower than the means of the respective variables in experiment 1 . However, these differences were not significant for any of the dependant variables.

Discussion of Experiment 3

The results of experiment 3 provide support for hypothesis 3. Assortments with alternatives differing in color were more attractive than those with options differing in form when the sets were large, while both were seen as equally appealing when set size was small. We suggest that the reason for the greater attractiveness of the assortments with items differing in color is the same as that discussed above. Assortments with alternatives differing in color have lower costs for the human visual system than sets with options varying in shape, thereby making the former more attractive than the latter.

The results of experiment 3 may have interesting implications for marketing. When offered two assortments - one with alternatives differing in color and the other in form - 
consumers may select the former and ignore the latter even when the most preferred option is available in the rejected set. Therefore, it is advisable to present wide assortments in different colors as these may attract more consumers than assortments with alternatives in different forms.

\section{GENERAL DISCUSSION}

This paper has explored the nature of satisfaction from choice as a function of characteristics of choice sets. Building upon the theoretical insights of Coombs and Avrunin (1977), we suggested that as the number of alternatives increases so do the resulting benefits and costs. However, whereas the former "satiate," the latter "escalate." The net effect is that satisfaction is an inverted U-shaped function of set size. Our laboratory experiments supported this proposition.

Visual presentation of choice sets also affects satisfaction. Because the costs of processing large assortments with alternatives differing in shape are greater than of those for options varying in color, we predicted and demonstrated that participants experience higher satisfaction when choosing from the latter. That is, the location of the peak of the satisfaction function is influenced by visual presentation of the assortment. The results also revealed that assortments with alternatives differing in color were not only more satisfying, but also more attractive for the customers.

Costs of information processing as well as benefits of choice may also depend on individual characteristics of consumers. In this regard we demonstrated that the satisfaction function was affected by cultural background of the participants. Although the satisfaction functions were shown to be of the inverted U-shape across both Eastern and Western European 
participants, the locations of the peaks differed. Participants from Eastern Europe were satisfied with larger assortments than those from Western Europe. In addition, in our experiments women appeared to be more satisfied with variety than men.

It is important to emphasize that whereas the general shape of the function is applicable across many circumstances, the specific optimal numbers of options found in our experiments can not be generalized since these depend on the perceived "costs" and "benefits" of each particular choice set. This position is, of course, entirely consistent with our theoretical analysis. In addition, our findings do not imply that shapes of alternatives have little value. These were shown to be important in small assortments. In addition, shapes can be of great value when customers discriminate and decide among alternatives, but not between assortments. This proposition, however, should be addressed in future research.

We now consider some limitations of the present research, suggestions for future research, and implications for marketing.

First, the participants in our experiments were given the goal to pick and "buy" a product. However, customers also visit shops without the intention to buy (Desmeules 2002) and may have the option "not to choose" (Dhar 1997). On the other hand, we note that in many situations customers have to make decisions sooner or later, even if these can be postponed. Whereas the satisfaction function investigated in this paper applies to situations when the choice is to be made, variations of the tasks we investigated could include, for example, studying the effects of time pressure, incidental examination of choices sets (i.e., with no or different purchasing aims), and deferral of choice. In all cases, we would expect the satisfaction function to be sensitive to the manner in which these variables affect perceived costs and benefits. 
Second, in our studies participants were not assumed to have established preferences over the gift boxes prior to viewing the assortments. However, if customers are loyal to a certain product, they may not care about the number of options available, and our results may not be applicable to such situations. Besides, if a consumer starts buying a certain type of item more often, she will obtain feedback and learn with time how to choose. That is, she will learn which attributes of the good she values more and thereby make better decisions and deal more easily with complexity. Experts in a given domain are characterized by the ability to process more relevant information than novices (see, e.g., Chase and Simon, 1973). We would therefore expect satisfaction functions to vary depending on consumers' knowledge and previous experience with the good.

Third, in this experimental work we simplified by focusing on simple objects that differed on only one or two attributes. Clearly, an important next step will be to extend the approach adopted here to more complex products in naturally-occurring field studies.

In addition to the above, our work suggests several lines of further research. First, the optimal number of alternatives (for satisfaction) in our studies was found to be 10 or 15 . These numbers are exactly the same as those reported by Miller (1956) for the "channel capacity" of visual positioning, that is, the number of visual positions the human eye can distinguish without making errors. It is unclear whether this is a coincidence. However, it suggests investigating whether satisfaction is an inverted U-shaped function of the number of alternatives when these are not characterized visually but by, say, tone, taste, or odor. Building upon our theoretical framework, we would still expect satisfaction to be an inverted U-shaped function of the numbers of tone, taste, or odor stimuli. As mentioned above, Miller (1956) argued that the "span of absolute judgment" is greater for visual stimuli than for tones or taste stimuli. Therefore, as 
the costs of processing the latter are higher, we would also expect the location of the peaks of the satisfaction functions for these to lie to the left of those for visual stimuli.

Second, the measures used in our studies were subjective reports of satisfaction. To assess the underlying costs of information processing better, it would be helpful to combine these reports with more objective measures obtained by, say, eye-tracking devices and, possibly, the techniques of neuroscience.

Third, the explanations of our findings were guided by our theoretical framework. Critical to this is the role of perceived costs and benefits. This therefore suggests the need for studies to examine these perceptions in depth.

Fourth, both in the current paper and previous research 30-option sets have been considered "large" and five-option sets "small." What, however, do consumers consider "large," "small," or "medium-sized" and how does this vary by types of products and consumers?

Fifth, we also found that large assortments with items differing in color were more attractive and appealing for participants than sets with options differing in shape. However, does it necessarily mean that these sets will be more "motivating," that is will customers purchase more from large sets with items differing in color than from those differing in form ? As Iyengar and Lepper (2000) have suggested, larger sets are both more attractive and less motivating. A similar situation could occur with the SCDF and SFDC assortments. It would be interesting to verify whether sets with alternatives differing in color are not only more attractive, but also more motivating.

Sixth, results showed that gender and culture may affect satisfaction from variety. However, our findings are only suggestive and whether they can be generalized across different conditions is an exciting issue for further research. 
Other potential topics include whether the optimal choice depends on the importance of the decision, whether consumers choose a good for a different person or for themselves, and how the relative importance of the different visual attributes - color versus form - affects satisfaction. As with most of the issues discussed above, we believe that our theoretical framework provides insights into how to investigate these issues.

The results of these experiments have implications for both the theory and practice of marketing. For example, the findings suggest that neither small nor large assortments are “optimal” for consumers. On average, consumers experience the highest level of satisfaction when offered medium-sized assortments which provide considerable variety but are not overwhelming.

The results also suggest that by creating "comfortable" visual environments firms can present even large assortments to consumers without decreasing their satisfaction. For example, if - in an advertisement - there is a wish to present many similar objects at the same time, it is more advisable to present these in different colors than in different forms. By doing so, advertisers may still offer a wide assortment without imposing high information processing costs on consumers. The same logic can be applied to the design of web-sites or layouts at exhibitions.

The results of the paper may also represent a cautionary note for international marketing. The finding that satisfaction from variety is culturally influenced suggests that whereas large offerings may be satisfying for some cultures, others should be offered less alternatives. Marketing strategies concerning variety cannot be assumed to be universal.

The knowledge of how much of the same good to offer and how to present variety can be beneficial both for retailers and customers. Well-designed assortments can induce positive affect among consumers, lead to greater satisfaction with the experience of shopping, and impact sales 
and profitability of retailers positively. Conversely, overloading consumers' cognitive capacities can have negative effects for all. Our experimental results suggest that knowledge of our theoretical insights could profit both consumers and marketers. 


\section{Appendix A}

\section{INCREASES/ DECLINES OF MEANS AMONG CHOICE SETS WITH DIFFERENT NUMBER OF ALTERNATIVES}

\begin{tabular}{|c|c|c|c|c|c|c|c|}
\hline Measure & Sample & 5 vs 10 & 5 vs 15 & 5 vs 30 & 10 vs 15 & 10 vs 30 & 15 vs 30 \\
\hline \multirow{2}{*}{$\begin{array}{l}\text { Satisfaction from } \\
\text { the gift box } \\
\text { picked }\end{array}$} & Experiment 1 & $+1.53 * * *$ & $+0.73 * *$ & +0.13 & $-0.80 * *$ & $-1.40 * * *$ & $-0.60 *$ \\
\hline & Experiment 2 & +0.14 & $+1.20 * * *$ & +0.60 & $+1.06 * *$ & +0.46 & -0.60 \\
\hline \multirow{2}{*}{$\begin{array}{l}\text { Perception of } \\
\text { similarity of the } \\
\text { box chosen to the } \\
\text { "ideal one" }\end{array}$} & Experiment 1 & $+1.5^{* * *}$ & +0.57 & +0.73 & $-0.93 *$ & -0.77 & +0.17 \\
\hline & Experiment 2 & +0.6 & $+1.97 * * *$ & $+1.03 *$ & $+1.37 * *$ & +0.43 & $-0.93 *$ \\
\hline \multirow{2}{*}{$\begin{array}{l}\text { Satisfaction from } \\
\text { the decision- } \\
\text { making process }\end{array}$} & Experiment 1 & $+1.37 * * *$ & $+1.23 * * *$ & $+0.97 * *$ & -0.14 & -0.40 & -0.26 \\
\hline & Experiment 2 & +0.37 & $+1.40^{* *}$ & +0.60 & $+1.03 *$ & +0.23 & -0.80 \\
\hline \multirow{2}{*}{ Difficulty level } & Experiment 1 & $+1.27 * *$ & $+1.27 * *$ & $+1.47 * *$ & 0 & +0.20 & +0.20 \\
\hline & Experiment 2 & $+1.70^{* *}$ & +0.27 & $+1.88 * * *$ & $-1.43 * *$ & +0.17 & $+1.6^{* *}$ \\
\hline
\end{tabular}

*** significant at 1 percent level

** significant at 5 percent level

*significant at 10 percent level 
Appendix B.

GENDER EFFECT FOR FOUR DEPENDENT VARIABLES

\begin{tabular}{|l|l|l|l|l||}
\hline \multirow{2}{*}{ Measure } & \multicolumn{2}{|c|}{ Gender dummy } & \multicolumn{2}{c|}{ Interaction options X gender } \\
\cline { 2 - 5 } & \multicolumn{1}{|c|}{ Experiment 1 } & Experiment 2 & Experiment 1 & Experiment 2 \\
\hline $\begin{array}{l}\text { Satisfaction from } \\
\text { the gift box }\end{array}$ & $\begin{array}{l}\mathrm{F}(1,115)=4.07 \\
\mathrm{p}=.046\end{array}$ & $\begin{array}{l}\mathrm{F}(1,115)=7.16 \\
\mathrm{p}=.009\end{array}$ & $\begin{array}{l}\mathrm{F}(3,112)=0.49 \\
\mathrm{p}=.693\end{array}$ & $\begin{array}{l}\mathrm{F}(3,112)=1.55 \\
\mathrm{p}=.206\end{array}$ \\
\hline $\begin{array}{l}\text { Perception of } \\
\text { similarity of the } \\
\text { box chosen to the } \\
\text { "ideal one" }\end{array}$ & $\begin{array}{l}\mathrm{F}(1,115)=6.15 \\
\mathrm{p}=.015\end{array}$ & $\begin{array}{l}\mathrm{F}(1,115)=0.50 \\
\mathrm{p}=.480\end{array}$ & $\begin{array}{l}\mathrm{F}(3,112)=0.69 \\
\mathrm{p}=.563\end{array}$ & $\begin{array}{l}\mathrm{F}(3,112)=0.60 \\
\mathrm{p}=.6163\end{array}$ \\
\hline $\begin{array}{l}\text { Satisfaction from } \\
\text { the decision- } \\
\text { making process }\end{array}$ & $\begin{array}{l}\mathrm{F}(1,115)=2.37 \\
\mathrm{p}=.013\end{array}$ & $\begin{array}{l}\mathrm{F}(1,115)=7.87 \\
\mathrm{p}=.006\end{array}$ & $\begin{array}{l}\mathrm{F}(3,112)=2.27 \\
\mathrm{p}=.084\end{array}$ & $\begin{array}{l}\mathrm{F}(3,112)=0.14 \\
\mathrm{p}=.935\end{array}$ \\
\hline $\begin{array}{l}\text { Difficulty level } \\
\mathrm{p}=.775\end{array}$ & $\mathrm{p}(1,115)=0.08$ & $\begin{array}{l}\mathrm{F}(1,115)=0.49 \\
\mathrm{p}=.487\end{array}$ & $\begin{array}{l}\mathrm{F}(3,112)=0.02 \\
\mathrm{p}=.997\end{array}$ & $\begin{array}{l}\mathrm{F}(3,112)=0.37 \\
\mathrm{p}=.774\end{array}$ \\
\hline $\begin{array}{l}\text { Perception of the } \\
\text { right number of } \\
\text { options }\end{array}$ & $\begin{array}{l}\mathrm{F}(1,115)=0.17 \\
\mathrm{p}=.683\end{array}$ & $\begin{array}{l}\mathrm{F}(1,115)=1.08 \\
\mathrm{p}=.302\end{array}$ & $\begin{array}{l}\mathrm{F}(3,112)=2.01 \\
\mathrm{p}=.117\end{array}$ & $\begin{array}{l}\mathrm{F}(3,112)=1.26 \\
\mathrm{p}=.290\end{array}$ \\
\hline \hline
\end{tabular}


Appendix C.

COMPLEXITY EFFECT FOR FOUR DEPENDENT VARIABLES

\begin{tabular}{|l|l|l|l|l|}
\hline \multirow{2}{*}{ Measure } & \multicolumn{2}{|c|}{ Complexity dummy } & \multicolumn{2}{c|}{ Interaction options X complexity } \\
\cline { 2 - 5 } & Experiment 1 & Experiment 2 & Experiment 1 & Experiment 2 \\
\hline $\begin{array}{l}\text { Satisfaction from } \\
\text { the gift box }\end{array}$ & $\begin{array}{l}\mathrm{F}(1,115)=9.81 \\
\mathrm{p}=.002\end{array}$ & $\begin{array}{l}\mathrm{F}(1,115)=5.72 \\
\mathrm{p}=.018\end{array}$ & $\begin{array}{l}\mathrm{F}(3,112)=1.14 \\
\mathrm{p}=.337\end{array}$ & $\begin{array}{l}\mathrm{F}(3,112)=2.72 \\
\mathrm{p}=.048\end{array}$ \\
\hline $\begin{array}{l}\text { Perception of } \\
\text { similarity of the } \\
\text { box chosen to the } \\
\text { "ideal one" }\end{array}$ & $\begin{array}{l}\mathrm{F}(1,115)=1.36 \\
\mathrm{p}=.246\end{array}$ & $\begin{array}{l}\mathrm{F}(1,115)=0.00 \\
\mathrm{p}=.953\end{array}$ & $\begin{array}{l}\mathrm{F}(3,112)=1.65 \\
\mathrm{p}=.182\end{array}$ & $\begin{array}{l}\mathrm{F}(3,116)=0.84 \\
\mathrm{p}=0.473\end{array}$ \\
\hline $\begin{array}{l}\text { Satisfaction from } \\
\text { the decision- } \\
\text { making process }\end{array}$ & $\begin{array}{l}\mathrm{F}(1,115)=3.34 \\
\mathrm{p}=.070\end{array}$ & $\begin{array}{l}\mathrm{F}(1,115)=0.07 \\
\mathrm{p}=.791\end{array}$ & $\begin{array}{l}\mathrm{F}(3,112)=0.18 \\
\mathrm{p}=.908\end{array}$ & $\begin{array}{l}\mathrm{F}(3,116)=0.26 \\
\mathrm{p}=.853\end{array}$ \\
\hline $\begin{array}{l}\text { Difficulty level } \\
\mathrm{p}=0.270\end{array}$ & $\begin{array}{l}\mathrm{F}(1,115)=1.23 \\
\mathrm{p}=0.878\end{array}$ & $\begin{array}{l}\mathrm{F}(3,112)=0.09 \\
\mathrm{p}=.966\end{array}$ & $\begin{array}{l}\mathrm{F}(3,116)=1.29 \\
\mathrm{p}=.282\end{array}$ \\
\hline $\begin{array}{l}\text { Perception of the } \\
\text { right number of } \\
\text { options }\end{array}$ & $\begin{array}{l}\mathrm{F}(1,115)=0.79 \\
\mathrm{p}=.377\end{array}$ & $\begin{array}{l}\mathrm{F}(1,115)=1.03 \\
\mathrm{p}=.312\end{array}$ & $\begin{array}{l}\mathrm{F}(3,112)=0.17 \\
\mathrm{p}=.915\end{array}$ & $\begin{array}{l}\mathrm{F}(3,116)=1.37 \\
\mathrm{p}=.256\end{array}$ \\
\hline
\end{tabular}




\section{References}

Carmon, Ziv, Klaus Wertenbroch, and Marcel Zeelenberg, M. (2003), “Option Attachment: When Deliberating Makes Choosing Feel Like Losing," Journal of Consumer Research, 30 (1): 15-29.

Chase, William .G. and Herbert A. Simon (1973), "Perception in chess," Cognitive Psychology, 4 (January): 55-81.

Coombs, Clyde and Avrunin, George (1977), "Single-peaked functions and the theory of preference," Psychological Review, 84 (2): 216-230.

Desmeules, Rémi (2002), “The Impact of Variety on Consumer Happiness: Marketing and the Tyranny of Freedom," Academy of Marketing Science Review [Online], 12; http://www.amsreview.org/articles/desmeules12-2002.pdf.

Dhar, Ravi (1997), “Context and Task Effects on Choice Deferral," Marketing Letters, 8 (1): 119-130.

Filin, Vasili. (2001), Videoecology: good and bad for the eye (In Russian), Moscow: MC Videoecology.

Huffman, Cynthia and Barbara E. Kahn (1998), "Variety for Sale: Mass Customization or Mass Confusion?” Journal of Retailing, 74 (4): 491-513.

Iyengar, Sheena and Mark R. Lepper (1999), "Rethinking the Value of Choice: A Cultural Perspective on Intrinsic Motivation," Journal of Personality and Social Psychology, 76 (3): 349-366.

(2000), "When choice is demotivating can one desire too much of a good thing?" Journal of personality and social psychology, 79 (6): 995-1006. 
Kahn, Barbara E. and Brian Wansink (2004), “The Influence of Assortment Structure on Perceived Variety and Consumption Quantities," Journal of Consumer Research, 30 (March): 519-533.

Loewenstein, George (1999), "Is more choice always better?" Social Security Brief: National Academy of Social Insurance, 7 (October): 1-8

Miller, George A. (1956), "The Magical Number Seven, Plus or Minus Two: Some Limits on Our Capacity for Processing Information,” The Psychological Review, 63: 81-97

Meyers-Levy, Joan (1998), "Mixed messages. How men and women differ in their responses to marketing messages," Capital ideas, 1(3): 7-8.

Meyers-Levy, Joan and Durairaj Maheswaran (1991), "Exploring differences in males' and females' processing strategies," Journal of Consumer Research, 18 (1): 63-70.

Rolls, Barbara J, Edward A. Rowe, Edmund T. Rolls, Breda Kindston, Angela Megson, and Rachel Gunary (1981), "Variety in a Meal Enhances Food Intake in Man,” Physiology and Behavior, 26 (February): 215-221.

Ryan, Richard M. and Edward L. Deci(2000), "Self-Determination Theory and the Facilitation of Intrinsic Motivation Social Development, and Well-Being," American Psychologist, 55 (1): 68-78.

Schwartz, Barry (2000). Self-determination: The tyranny of freedom. American Psychologist, 55 (1): 79-88.

(2004). The paradox of choice: why more is less, New York: Ecco. 
Schwartz, Barry, Andrew Ward, Darrin R. Lehman, John Monterosso, Katherine White, and Sonja Lyubomirsky (2002), "Maximizing versus satisficing: Happiness is a matter of choice," Journal of Personality and Social Psychology, 83 (5): 1178-1197

Shafir, Eldar, Amos Tversky, and Itamar Simonson (1993), "Reason-based choice," Cognition, 49(1-2): 11-36

Spring, Michael and Michael C. Jennings (1993), "Virtual Reality and Abstract Data: Virtualizing Information," Virtual Reality World, 1 (1)

Zuckerman, Miron, Drew Lathin, Edward L Deci, Joseph Porac, and, Raymond Smith (1978), "On the importance of self-determination for intrinsically motivated behavior," Personality and Social Psychology Bulletin, 4 (July): 443-446. 
Tables and Figures

TABLE 1

SIGNIFICANCE OF THE ASSORTMENT SIZE EFFECT ON DEPENDENT VARIABLES

\begin{tabular}{|c|c|c|}
\hline \multirow[b]{2}{*}{ Dependent variable } & \multicolumn{2}{|c|}{ Statistics } \\
\hline & Experiment 1 & Experiment 2 \\
\hline $\begin{array}{l}\text { Satisfaction from the gift box } \\
\text { picked }\end{array}$ & $\begin{array}{l}F(3,116)=8.92 \\
p=.000\end{array}$ & $\begin{array}{l}F(3,116)=3.35 \\
p=.022\end{array}$ \\
\hline $\begin{array}{l}\text { Satisfaction from the decision } \\
\text { process }\end{array}$ & $\begin{array}{l}F(3,116)=4.07 \\
p=.009\end{array}$ & $\begin{array}{l}F(3,116)=2.22 \\
p=.089\end{array}$ \\
\hline $\begin{array}{l}\text { Perception of the right number } \\
\text { of options }\end{array}$ & $\begin{array}{l}F(3,116)=10.21 \\
p=.000\end{array}$ & $\begin{array}{l}F(3,116)=2.78 \\
p=.044\end{array}$ \\
\hline $\begin{array}{l}\text { Perception of similarity of the } \\
\text { box chosen to the "ideal one" }\end{array}$ & $\begin{array}{l}F(3,116)=3.06 \\
p=.031\end{array}$ & $\begin{array}{l}F(3,116)=4.43 \\
p=.006\end{array}$ \\
\hline Difficulty & $\begin{array}{l}F(3,116)=2.77 \\
p=.045\end{array}$ & $\begin{array}{l}F(3,116)=4.41 \\
p=.006\end{array}$ \\
\hline
\end{tabular}




\section{FIGURE 1}

SATISFACTION AS A FUNCTION OF THE NUMBER OF ALTERNATIVES

FIGURE 1A

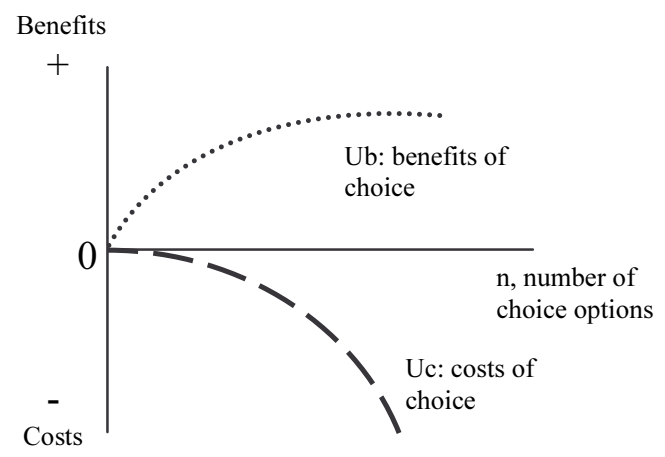

FIGURE 1B

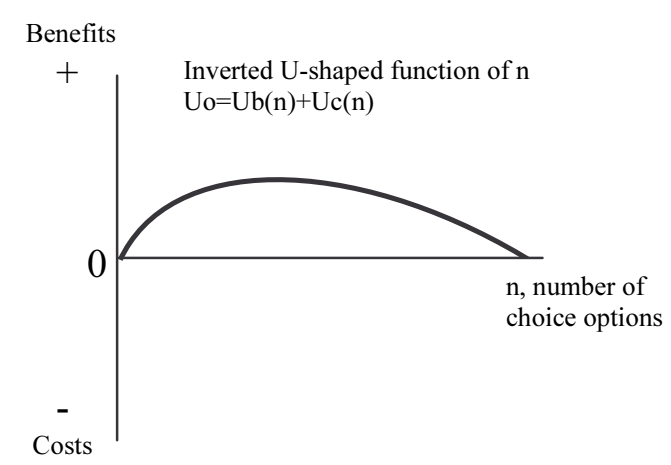


FIGURE 2

SATISFACTION FUNCTION FROM THE GIFT BOX PICKED

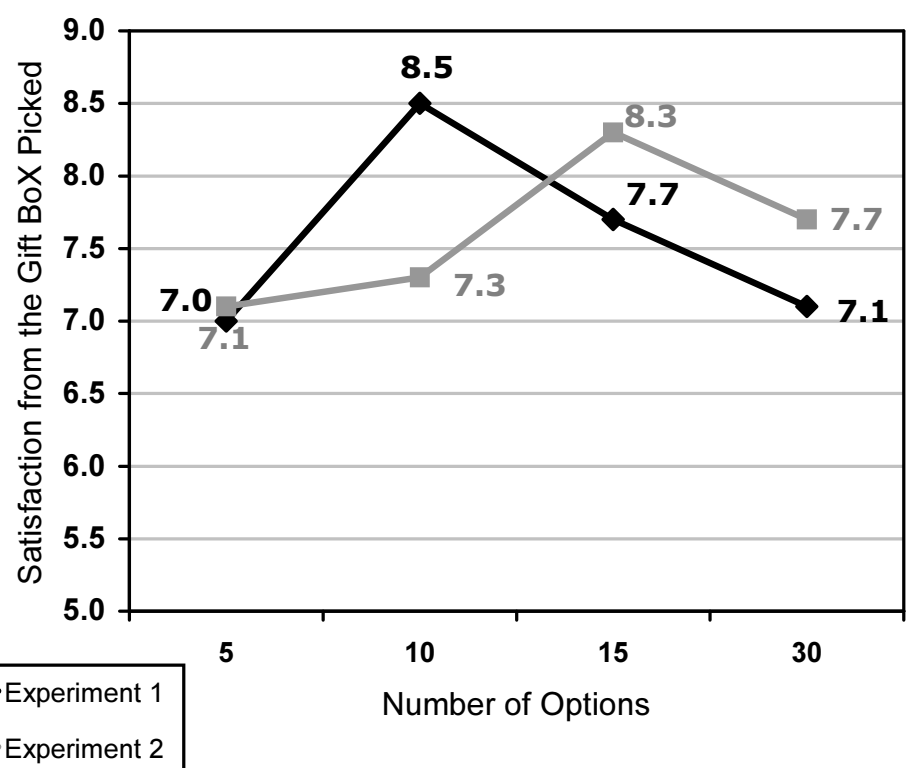


FIGURE 3

PERCEPTION OF SIMILARITY OF THE BOX CHOSEN TO THE "IDEAL ONE"

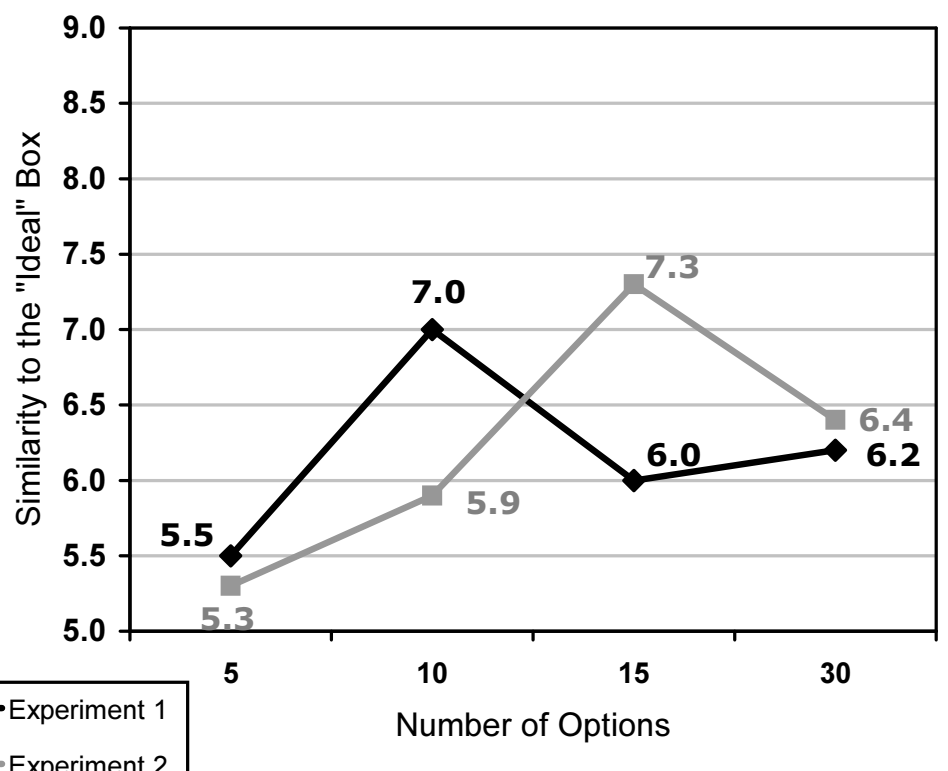


FIGURE 4

SATISFACTION FROM THE DECISION-MAKING PROCESS

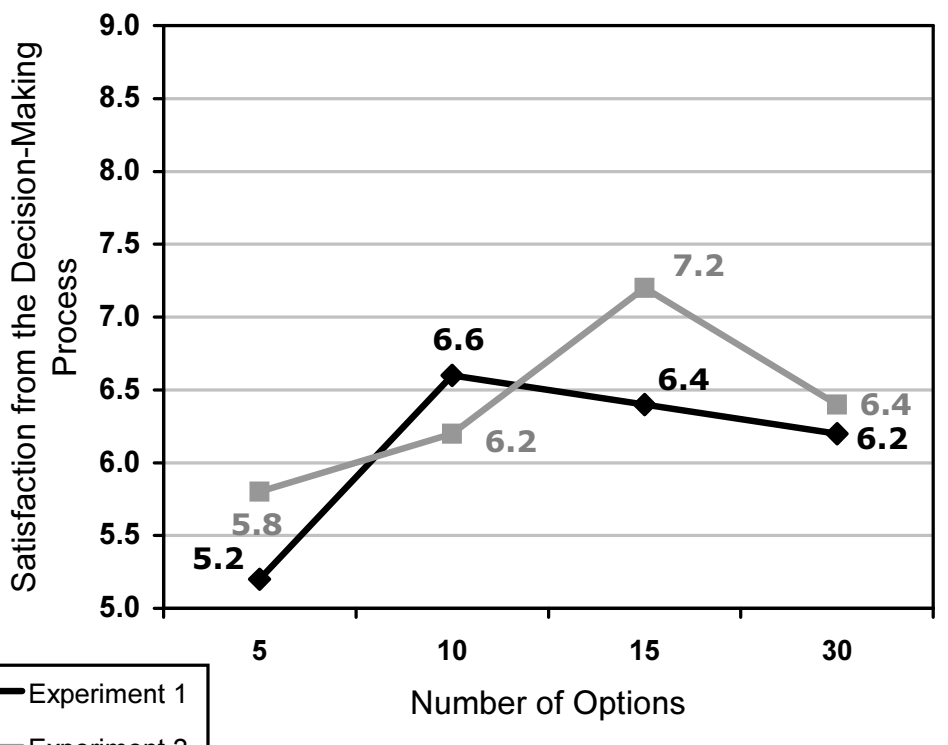


FIGURE 5

DIFFICULTY LEVEL

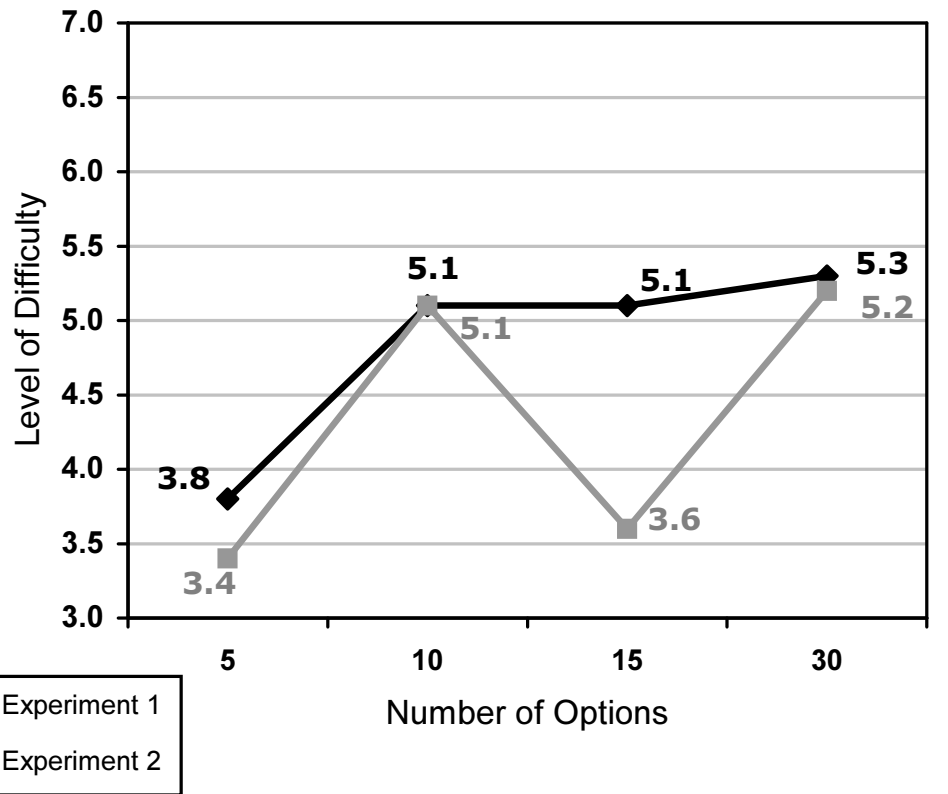


FIGURE 6

PERCEPTION OF THE “RIGHT NUMBER” OF OPTIONS IN THE CHOICE SET

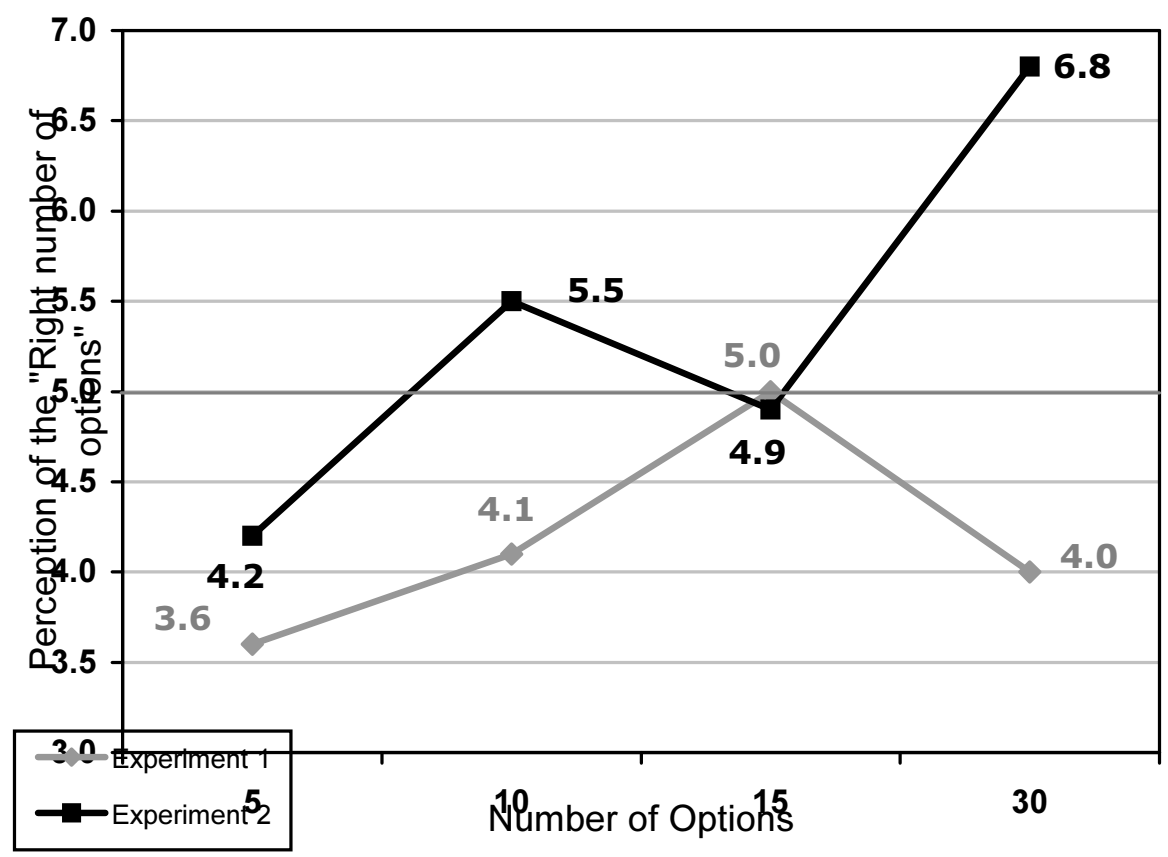


FIGURE 7

\section{EFFECT OF DIFFERENT VISUAL PRESENTATION, EXPERIMENT 1}

FIGURE 7A

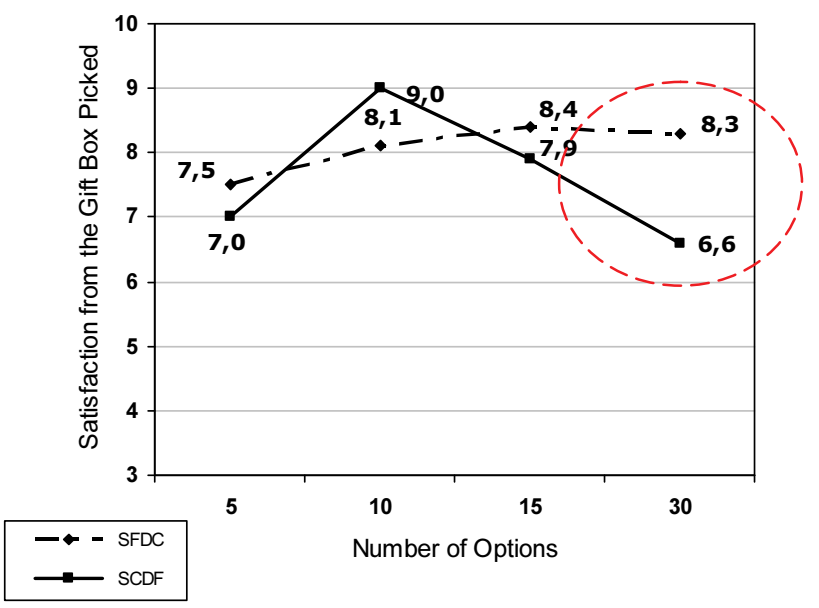

FIGURE 7C

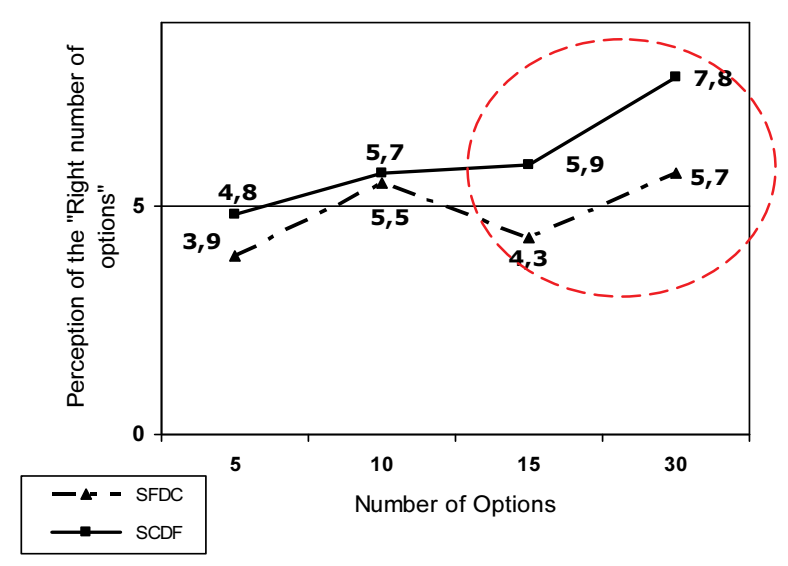

FIGURE 7B

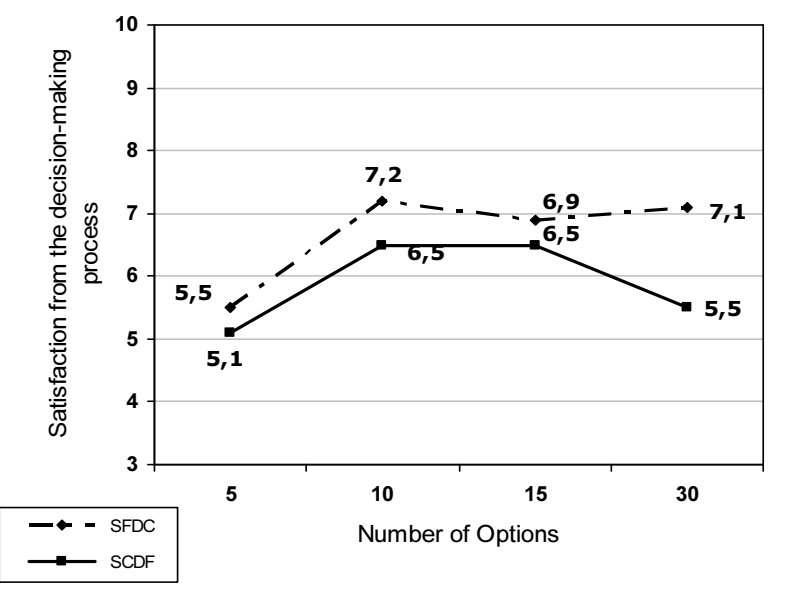


FIGURE 8

EFFECT OF DIFFERENT VISUAL PRESENTATION, EXPERIMENT 2

FIGURE 8A

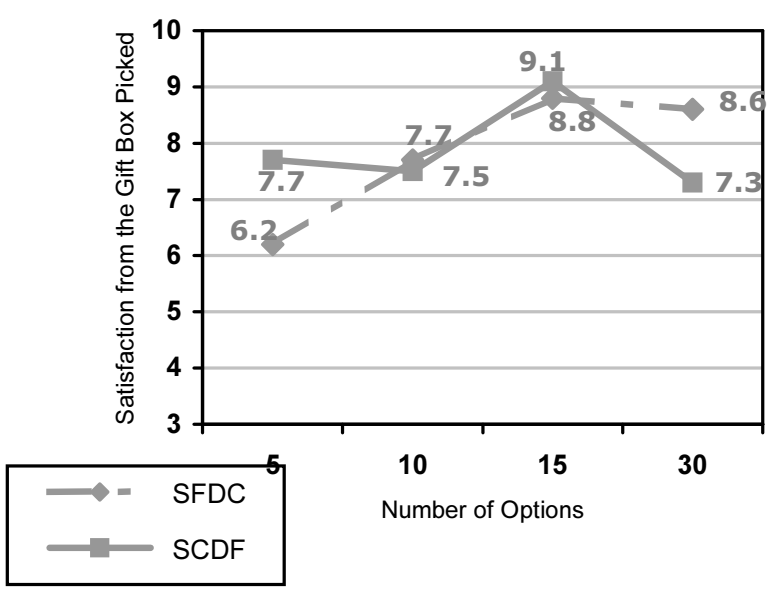

FIGURE 8C

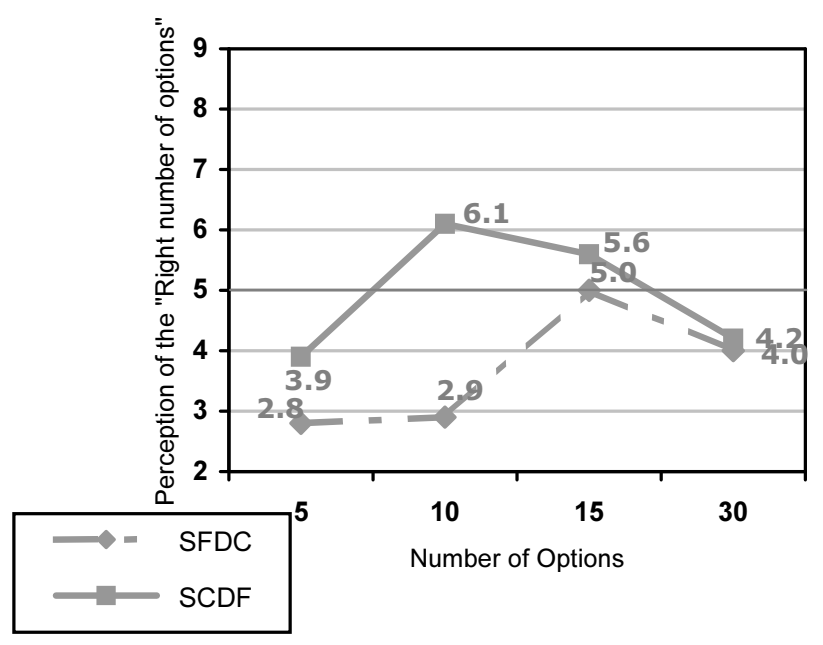

FIGURE 8B

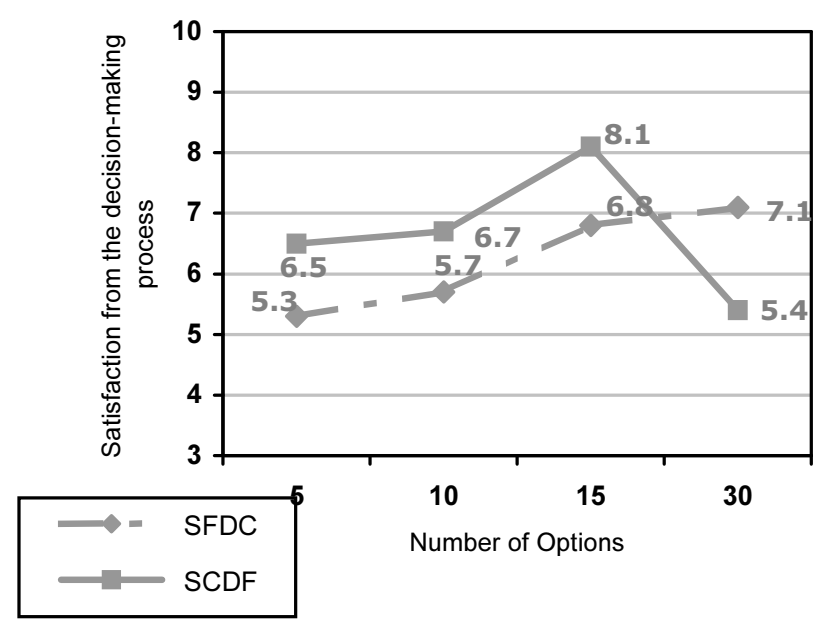

